\title{
OPTIMAL DECISION MAKING FOR AIRLINE INVENTORY CONTROL
}

\author{
Ioana C. Bilegan ${ }^{1,2}$, Sergio González-Rojo ${ }^{1,3}$, Carlos A.N. Cosenza ${ }^{4}$, Félix \\ Mora-Camino ${ }^{1,2}$ \\ ${ }^{\prime} L A A S$-CNRS, Toulouse, France; ${ }^{2} E N A C / D G A C$, Air Transportation Department, Toulouse, \\ France; ${ }^{3}$ ITCH, Chihuahua, Mexico; ${ }^{4}$ COPPE/UFRJ, Rio de Janeiro, Brasil.
}

\begin{abstract}
This paper presents a market-reactive optimization approach to solve, on-line, the airline Revenue Management problem. The recursive demand forecasting method proposed, which makes use of geometric programming is described and the uncertainties related to the demand arrival process are taken into account within the Inventory Control module via the use of stochastic dynamic programming. A new backward recursive dynamic programming model is developed and implemented for different situations of fare-classes confinement and numerical results and performance assessments are obtained by computer simulations.
\end{abstract}

Key words: optimization; decision support systems; dynamic systems; market-reactive revenue management; demand forecasts; decision making under uncertainty; probabilistic model; geometric programming; dynamic programming.

\section{INTRODUCTION}

The aim of airline Revenue Management systems is to contribute to the efficiency of airline companies by maximizing their revenues obtained from selling the available seats on the flights they offer (fixed amounts of perishable services), based on reliable demand forecasts.

Following McGill and van Ryzin (1999), all revenue management systems can be considered to have four main components: forecasting, overbooking, seat inventory control and pricing. All those elements are important by themselves, but a natural hierarchy exists between them since, for example, the seat inventory control mechanism drives what optimization algorithms can be employed and the inputs needed by the optimization algorithm drive the demand model and what types of forecasts are needed. 
This new management technique revolutionized airline industry and many other perishable-asset industries.

A reservation process has to be controlled and implemented within a complex decision support environment which should be able to make, online, the right decision with respect to any booking request received by the Computer Reservation System (CRS) at any point in time during the booking horizon.

In the proposed approach, the optimization module of the Revenue Management system works on-line, it gathers as input all the most recent updates provided by a demand forecasting function, as well as the present state of the reservations, to proceed with an optimization algorithm for the booking control process which treats new requests.

In order to take into account the highly stochastic nature of booking requests, forecasts should be updated with the latest information available. Therefore, a feedback control loop can be established between the Inventory Control module and the Demand Forecasting Updating process, leading to a market reactive Revenue Management system. The structure of the proposed system is then such as presented in Figure 1.

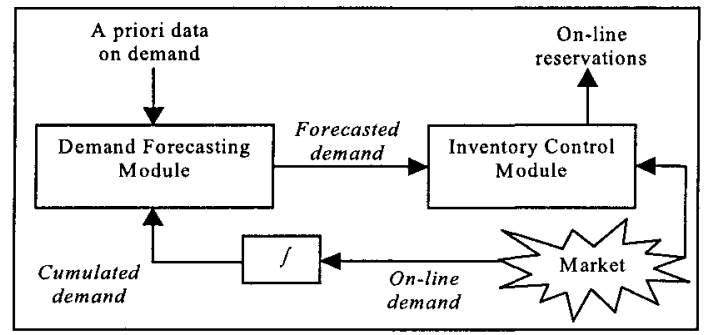

Figure 1. Simplified structure for airline RM systems.

\section{PROBABILITIES UPDATING METHOD}

The temporal (daily) dimension of the reservation process in the airline industry has to be taken into account for a realistic implementation of any optimization method. The Dynamic Programming technique, whose theoretical foundations were established in the early fifties, is used now in air transportation field with increased efficiency. Two main reasons can be quoted for that: first, the drastic increase in computational power of recent computers; second, the development of new forecasting methods which allow to estimate more accurately the probabilistic parameters describing the booking requests arrival process, which are needed by the Dynamic Programming algorithm. 
In order to develop a stochastic Dynamic Programming approach to manage requests for travel occurring during predefined time periods, it is necessary to have at hand a representative probability distribution of demand by period, by fare-class and by order of arrival. Considering $p_{i, m}^{n}$ as the probability to have $m$ demands for the class $i$ during the decision period $n$, it is possible, for independent booking requests arrivals, to define the probability $\wp^{n}{ }_{i, k}$ to have during decision period $n$, a $k^{\text {th }}$ demand for fareclass $i$ :

$$
\wp_{i, k}^{n}=\left(\sum_{l=0}^{K-k} \sum_{m_{1}+\cdots+m_{l}=k+l} \prod_{i=1}^{I} p_{i, m_{i}}^{n}\right) \cdot \frac{\sum_{m=0}^{K_{i}} m \cdot p_{i, m}^{n}}{\sum_{i=1}^{I} \sum_{m=0}^{K_{i}} m \cdot p_{i, m}^{n}}
$$

with $K=K_{I}+K_{2}+\ldots+K_{I}, K_{i}$ being the maximum number of demands for fareclass $i$ during decision period $n$ and $I$ the number of fare-classes.

Relation (1) is based on the distribution of the $p_{i, m}^{n}$, which is the probability to have $m$ demands for the class $i$ during the decision period $n$. This distribution can be made available by a demand forecasting module and can be updated daily, based on the latest information (newly registered bookings) available to the Revenue Management system.

In the proposed approach, the updating process of the $p^{n}{ }_{j k}$ probabilities of upcoming demand makes use of a dual geometric programming formulation (Mora-Camino, 1978) for an optimization criterion of the information gain type (Jumarie, 1990):

$$
\max \sum_{k=n+1}^{N} \sum_{j=0}^{J} \frac{p_{j k}^{n}}{N-n} \cdot \log \left(\frac{p_{j k}^{n-1}}{p_{j k}^{n}}\right)
$$

under positivity (3), normality (4) and orthogonality $(5,6)$ constraints:

$$
\begin{aligned}
& \frac{p_{j k}^{n}}{N-n} \geq 0 \\
& \sum_{k=n+1}^{N} \sum_{j=0}^{J} \frac{p_{j k}^{n}}{N-n}=1
\end{aligned}
$$




$$
\begin{aligned}
& \sum_{k=n+1}^{N} \sum_{j=0}^{J}\left(j-\frac{\delta_{n}}{N-n}\right) \cdot p_{j k}^{n}=0 \\
& \sum_{j=0}^{J} p_{j(n+1)}^{n}-\sum_{j=0}^{J} p_{j k}^{n}=0
\end{aligned}
$$

where $k \in\{n+2, \mathrm{n}+3, \ldots, N\}, n$ is the decision period, $j$ is the number of demands and $p^{n-1}{ }_{j k}$ are the initial probability distributions, before updating. For more details and notations, see Bilegan, et al. (2001).

Once the problem is transformed into a non-constrained non-convex minimization problem, making use of the corresponding primal form of the geometric program, relation (7), genetic algorithms are used to solve it.

$$
\min \phi(\bar{t})=\sum_{j=0}^{J} p_{j(n+1)}^{n-1} \cdot t_{n+1}^{j-\frac{\delta_{n}}{N-n}} \cdot \prod_{k=n+2}^{N} t_{k}+\sum_{k=n+2}^{N} \sum_{j=0}^{J} p_{j k}^{n-1} \cdot t_{n+1}^{j-\frac{\delta_{n}}{N-n}} \cdot t_{k}^{-1}
$$

with $t_{k}>0$, for $k \in\{n+1, n+2, \ldots, N\}$.

The reasons of choosing genetic algorithms optimization technique (Goldberg, 1989) are given by the simplicity of encoding and the rapidness of global solution finding of the minimum of the non-convex continuous function $\phi(t)$, which is the objective of the associated primal geometric program.

Then, using geometric duality relations, the updated probability distributions will be finally computed and made available for further use, according with relations (8) and (9):

for $k=n+1$ :

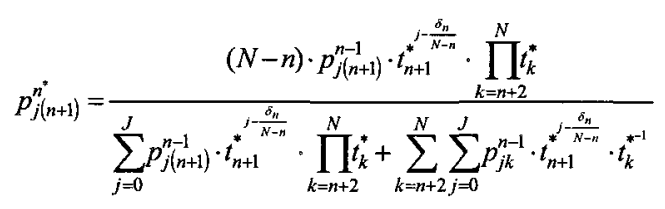

for $k=n+2$ à $N$ : 


$$
p_{j k}^{n^{*}}=\frac{(N-n) \cdot p_{j k}^{n-1} \cdot t_{n+1}^{*-\frac{\delta_{n}}{N-n}} \cdot t_{k}^{*-1}}{\sum_{j=0}^{J} p_{j(n+1)}^{n-1} \cdot t_{n+1}^{*-\frac{\delta_{n}}{N-n}} \cdot \prod_{k=n+2}^{N} t_{k}^{*}+\sum_{k=n+2}^{N} \sum_{j=0}^{J} p_{j k}^{n-1} \cdot t_{n+1}^{*-\frac{\delta_{n}}{N-n}} \cdot t_{k}^{*-1}}
$$

\section{DECISION MAKING USING DYNAMIC PROGRAMMING}

For the inventory control part of the proposed approach, the probability distributions obtained with the forecast updating module described in Section 2 are used as input data for the implementation of a Dynamic Programming optimization module.

The technique of Dynamic Programming has been established by Bellman (1957) to cope with sequential dynamic optimization problems with applications in many different fields: Optimal Control (Bertsekas, 2000; Bertsekas, 2001), Operations Research (Hillier and Lieberman, 1967; Winston, 1994), Management Sciences (Fabrycky and Torgersen, 1966). Some authors (Lee and Hersh, 1993; Subramanian, 1995; Talluri and van Ryzin, 1998) have already made use of this technique, in a limited way, to cope with sequential decision process in airline Revenue Management.

In the previously published works about the application of Dynamic Programming to treat booking requests arrivals (Gerchak, et al., 1985), the following assumption was always made: in each decision period there is at most one booking request and the considered length of the successive decision periods must be such that this assumption holds. This approach has been accepted until recently on theoretical grounds, but it cannot be translated exactly into an on-line discrete decision process.

Therefore, in this communication, the proposed approach is such that the updating of demand forecasts is treated on a daily basis, turning the whole process of easy implementation. The main idea behind this proposal is the assumption that the forecaster has a better capability to perform accurate predictions over relatively large and fixed length time intervals (i.e. 24 hours, the natural daily time-frame of a booking process) rather than on variable and generally short-time decision periods.

The inventory control problem is solved here via a daily based Dynamic Programming model, developed for two distinct situations: physically unconfined and physically confined fare-classes. The following main assumptions have to be made for the two situations:

- the demand probability distributions for the different fare-classes are considered to be completely independent;

- only single leg flights are considered, cancellations and no-shows are not taken into account; 
- go-shows are implicitly accounted for since bookings are permitted until the last moment before boarding closure; it is also implicitly considered that an estimate of the probability distributions for the "last day" demands (so, including the go-show probability distributions) are available;

- the whole capacity of the aircraft $(C)$ forms a pool of seats available for reservations in all the fare-classes offered by the airliner on a single leg flight (no physically confined classes);

- the dynamic booking limits (changing with time/decision period and remaining available capacity) for each fare-class are made available by the solution of the expected revenue maximization problem;

- a decision period $n, n \in\{N, N-1, \ldots, 0\}$, lasts 24 hours (bookings by Internet can be performed at any time during the day);

- during each decision period $n$, a limited number of demand requests $\left(\min =0, \max =K_{i}\right)$ for each of the fare-classes, $i \in\{1,2 \ldots, I\}$, can be received by the reservation system;

- a booking request can be either accepted or rejected; if rejected, it is considered lost for the company (no recapture possibility is integrated in the model, the probability of buy-up is not quantified);

and according to the following notations:

- $n=N$ denotes the "first booking day" (the first decision period) of booking process for a given scheduled flight;

- $n=1$ denotes the "last booking day", i.e. the day of departure, before boarding closure of a given scheduled flight;

- $n=0$ denotes the period following the boarding closure before flight departure and during which no revenue can be achieved any more, regardless the number of seats still available; so the initial conditions for the recursive Dynamic Program will be indexed by $n=0$, as shown in relation (14).

\subsection{Unconfined fare-classes}

The first situation studied here considers the case in which the entire pool of seats of the aircraft cabin is available for bookings for all fare-classes offered on a flight-leg.

Let $\varphi_{s}^{n, k}$ be the maximum expected revenue to be obtained from the booking process when $s$ seats are still available for booking and $(K-k)$ requests have been already made during the $n^{\text {th }}$ decision period. Then, the recursive expressions of the formulation of the backward Dynamic Programming model are given in relations (10) and (11): 


$$
\begin{aligned}
& \varphi_{s}^{n, k}=\left(1-\sum_{l=0}^{K-k} \sum_{m_{1}+m_{2}+\cdots+m_{l}=k+l} \prod_{i=1}^{I} p_{i, m_{i}}^{n}\right) \cdot \varphi_{s}^{n, k-1} \\
& +\sum_{i=1}^{I} \wp_{i, k}^{n} \cdot \max \left\{F_{i}+\varphi_{s-1}^{n, k-1}, \varphi_{s}^{n, k-1}\right\} \\
& \forall \mathrm{n} \in\{\mathrm{N}, \mathrm{N}-1, \ldots, 1\} \text { and } \forall \mathrm{s} \in\{0,1, \ldots, \mathrm{C}\} \\
& \varphi_{s}^{n, 0}=\varphi_{s}^{n-1, K}
\end{aligned}
$$

The decision criterion to accept or to reject an individual booking request is then given by:

$$
F_{i}+\varphi_{s-1}^{n, k-1} \geq \varphi_{s}^{n, k-1}
$$

This condition is based on the expected revenue maximization idea (Littlewood, 1972; Belobaba, 1989), which consists in accepting a booking request for a fare-class $i$, when $s$ seats are still available for booking, only if the immediate revenue obtained from this $\left(F_{i}\right)$ plus the maximum expected revenue to be obtained from the remaining available capacity $(s-1)$ is greater or equal to the maximum expected revenue to be obtained if the actual booking request was refused (i.e. for future bookings, $s$ seats would still be available).

In the case of group booking requests, this formula becomes:

$$
q \cdot F_{i}+\varphi_{s-q}^{n, k-q} \geq \varphi_{s}^{n, k-q}
$$

where $q$ is the size of the group.

The initial conditions to compute the maximum expected revenue are such as:

$$
\varphi_{s}^{0, k}=0
$$

for $k \in\{1, \ldots, \mathrm{K}\}$ and $s \in\{0,1, \ldots, \mathrm{C}\}$.

\subsection{Physically confined fare-classes}

The above model is extended here to the case where the aircraft cabin is divided in two different spaces, Business and Economy fareclasses, which have separate seat availability on the same flight-leg. Additional assumptions are necessary: 
- a booking request for a seat in the Business Class can be either accepted, if there are seats available in the Business Class, or rejected (a business passenger cannot be accommodated on an economy seat - a lower standing);

- a booking request for a seat in the Economy Class can be accepted in the Economy Class, if there are seats available and can be also accepted in the Business Class if there are seats available in this class and no seats available in the Economy Class, or rejected (an economy passenger can be accommodated on a business seat - a higher standing);

and additional notations are adopted:

- the Business Class has a total capacity of $C_{b}$ seats;

- $\sigma$ is the number of seats still available for booking in Business Class;

- the Economy Class has a total capacity of $C_{e}$ seats;

- $s$ is the number of seats still available for booking in Economy Class;

- the total capacity of the aircraft is $C=C_{b}+C_{e}$;

- the business fare-classes are denoted by $\mathrm{i}, i \in\left\{1,2, \ldots, I_{b}\right\}$;

- the maximum number of booking requests that can arrive in Business Class during one decision period is given by $K_{\mathrm{b}}=K_{1}+K_{2}+\ldots+K_{\mathrm{Ib}}$;

- the economy fare-classes are denoted by $i, \mathrm{i} \in\left\{I_{b}+1, I_{b}+2, \ldots, I_{b}+I_{e}\right\}$;

- the maximum number of booking requests that can arrive in Economy Class during one decision period is given by $K_{\mathrm{e}}=K_{\mathrm{lb}+1}+K_{\mathrm{lb}+2}+\ldots+K_{\mathrm{lb}+\mathrm{le}}$;

- in total, there are $I=I_{b}+I_{e}$ fare-classes offered on the aircraft.

- The formulation of a decision process based on Dynamic Programming for booking control is in this case more complex, since passengers can be assigned to two different pulls of seats.

Let $\varphi_{s, \sigma}^{n, k, j}$ be the maximum expected revenue when $s$ seats are still available in the Economy Class, $\sigma$ seats are still available in the Business Class, when $\left(K_{e}-k\right)$ Economy Class booking requests and $\left(K_{b}-j\right)$ Business Class booking requests have been already made during the $n^{\text {th }}$ decision period.

The recursive expressions of the formulation of the backward Dynamic Program in this second case are now given by: 


$$
\begin{aligned}
& \varphi_{s, \sigma}^{n, k, j}=\left(1-\sum_{l=0}^{K_{e}-k} \sum_{m_{l_{b}+1}+\cdots+m_{l_{b}+l_{e}}=k+l} \prod_{i=1}^{I_{b}+I_{e}} p_{i, m_{i}}^{n}\right) \\
& \cdot\left(1-\sum_{d=0}^{K_{b}-j} \sum_{m_{1}+\cdots+m_{l_{b}}=j+d} \prod_{i=1}^{I_{b}} p_{i, m_{i}}^{n}\right) \cdot \varphi_{s, \sigma}^{n, k-1, j-1} \\
& +\sum_{i=I_{b}+1}^{I_{b}+I_{e}} \wp_{i, k+j}^{n} \cdot \max \left\{F_{i}+\varphi_{s-1, \sigma}^{n, k-1, j}, F_{i}+\varphi_{s, \sigma-1}^{n, k-1, j}, \varphi_{s, \sigma}^{n, k-1, j}\right\} \\
& +\sum_{i=1}^{I_{b}} \wp_{i, k+j}^{n} \cdot \max \left\{F_{i}+\varphi_{s, \sigma-1}^{n, k, j-1}, \varphi_{s, \sigma}^{n, k, j-1}\right\}
\end{aligned}
$$

with $n \in\{N, N-1, \ldots, 1\}, s \in\left\{0,1, \ldots, C_{e}\right\}, \sigma \in\left\{0,1, \ldots, C_{b}\right\}, k \in\{1$, $\left.2 \ldots, K_{e}\right\}, j \in\left\{1,2, \ldots, K_{b}\right\}$

and by:

$$
\varphi_{s, \sigma}^{n, 0,0}=\varphi_{s, \sigma}^{n-1, K_{e}, K_{b}}
$$

The initial conditions, adapted from the previous case, are such as:

$$
\varphi_{s, \sigma}^{0, k, j}=0
$$

The decision criterion to accept or reject an individual booking request in the Economy Class is in this case obtained by analyzing two possible situations.

If there are still some seats available in the Economy Class, the accepting condition established in the previous section can be adapted, from relation (12), for the pull of seats of the Economy Class; the $\left(K_{e}-k+I\right)^{\text {th }}$ booking request becomes an Economy Class reservation if:

$$
F_{i}+\varphi_{s-1, \sigma}^{n, k-1, j} \geq \varphi_{s, \sigma}^{n, k-1, j}
$$

To cope with the case where all the seats of the Economy Class are already booked while seats remain available in the Business Class, the accepting condition for an Economy Passenger on a Business Seat can be adapted from relation (12); then, the $\left(K_{e}-k+1\right)^{\text {th }}$ booking request becomes an Economy Class reservation if: 


$$
F_{i}+\varphi_{s, \sigma-1}^{n, k-1, j} \geq \varphi_{s, \sigma}^{n, k-1, j}
$$

For a booking request in the Business Class, there is only one case that must be analyzed, since these booking requests cannot be accommodated elsewhere than in the pull of seats of the Business Class. If there are still some seats available in this class, the accepting condition established in the previous section can also be adapted, from relation (12), for the pull of seats of the Business Class. The $\left(K_{b}-j+l\right)^{\text {th }}$ booking request becomes a Business Class reservation if:

$$
F_{i}+\varphi_{s, \sigma-1}^{n, k, j-1} \geq \varphi_{s, \sigma}^{n, k, j-1}
$$

Otherwise, booking requests (either in Economy Class of Business Class) which cannot be satisfied by applying these conditions will be denied.

\section{COMPUTATIONAL RESULTS}

In this section a numerical example provides results obtained by the direct implementation of the backward Dynamic Programming model of the inventory control module, for physically unconfined fare-classes, presented in Section 3.

The simulation example consists in a single-leg flight from origin A to destination $\mathrm{B}$, with three fare-classes $\left(F_{1}>F_{2}>F_{3}\right)$. The available capacity, 4 days before departure, is considered to be 20 seats. The maximum number of daily booking requests to come per fare-class is taken equal to 5 , and thus the total maximum number of demands per decision period is considered to be 15 .

The input data of the Dynamic Program consists of the probability distributions provided by the demand forecasting model which was described in Section 2, i.e. the direct application of relations (8) and (9) to solutions obtained using a classical (binary encoding) genetic algorithm optimization technique. For each of the three fare-classes, a different implementation, with different initial conditions for the genetic algorithm was used, such that for each fare-class different probability distributions, related to the value of the corresponding fare (the fare-class mean demand decreasing with $F_{i}$ ) were made available.

In Figure 2 (for fare-class 1), Figure 3 (for fare-class 2) and Figure 4 (for fare-class 3 ) the decision matrices obtained by using the proposed Dynamic Programming approach are presented. The example, which is representative for a high demand scenario (the total number of available seats is inferior to 
the total number of booking requests), corresponds to the decision period $n=2$ days before departure, where "*" means acceptance and "-" means denial of the corresponding booking request (when maximum 20 seats are still available for bookings and maximum 15 demands have already arrived to the CRS during the decision period of one day).

The performances of the decision making strategy obtained via the proposed Dynamic Programming model are evaluated by comparing the results of its implementation with the ones of the first come first served (FCFS) algorithm, applied to the same set of simulation data.

The comparative results presented in Figure 5 and Figure 6 demonstrate the superiority of the Dynamic Programming proposed approach.

\begin{tabular}{lllllllllllllllll}
$20:$ & $*$ & $*$ & $*$ & $*$ & $*$ & $*$ & $*$ & $*$ & $*$ & $*$ & $*$ & $*$ & $*$ & $*$ & $*$ & 15 \\
$19:$ & $*$ & $*$ & $*$ & $*$ & $*$ & $*$ & $*$ & $*$ & $*$ & $*$ & $*$ & $*$ & $*$ & $*$ & $*$ & - \\
$18:$ & $*$ & $*$ & $*$ & $*$ & $*$ & $*$ & $*$ & $*$ & $*$ & $*$ & $*$ & $*$ & $*$ & $*$ & $*$ & - \\
$17:$ & $*$ & $*$ & $*$ & $*$ & $*$ & $*$ & $*$ & $*$ & $*$ & $*$ & $*$ & $*$ & $*$ & $*$ & $*$ & - \\
$16:$ & $*$ & $*$ & $*$ & $*$ & $*$ & $*$ & $*$ & $*$ & $*$ & $*$ & $*$ & $*$ & $*$ & $*$ & $*$ & - \\
15 & $*$ & $*$ & $*$ & $*$ & $*$ & $*$ & $*$ & $*$ & $*$ & $*$ & $*$ & $*$ & $*$ & $*$ & $*$ & - \\
$14:$ & $*$ & $*$ & $*$ & $*$ & $*$ & $*$ & $*$ & $*$ & $*$ & $*$ & $*$ & $*$ & $*$ & $*$ & $*$ & - \\
13 & $*$ & $*$ & $*$ & $*$ & $*$ & $*$ & $*$ & $*$ & $*$ & $*$ & $*$ & $*$ & $*$ & $*$ & $*$ & - \\
12 & $*$ & $*$ & $*$ & $*$ & $*$ & $*$ & $*$ & $*$ & $*$ & $*$ & $*$ & $*$ & $*$ & $*$ & $*$ & - \\
11 & $*$ & $*$ & $*$ & $*$ & $*$ & $*$ & $*$ & $*$ & $*$ & $*$ & $*$ & $*$ & $*$ & $*$ & $*$ & - \\
10 & $*$ & $*$ & $*$ & $*$ & $*$ & $*$ & $*$ & $*$ & $*$ & $*$ & $*$ & $*$ & $*$ & $*$ & $*$ & - \\
$9:$ & $*$ & $*$ & $*$ & $*$ & $*$ & $*$ & $*$ & $*$ & $*$ & $*$ & $*$ & $*$ & $*$ & $*$ & $*$ & - \\
$8:$ & $*$ & $*$ & $*$ & $*$ & $*$ & $*$ & $*$ & $*$ & $*$ & $*$ & $*$ & $*$ & $*$ & $*$ & $*$ & - \\
$7:$ & $*$ & $*$ & $*$ & $*$ & $*$ & $*$ & $*$ & $*$ & $*$ & $*$ & $*$ & $*$ & $*$ & $*$ & $*$ & - \\
$6:$ & $*$ & $*$ & $*$ & $*$ & $*$ & $*$ & $*$ & $*$ & $*$ & $*$ & $*$ & $*$ & $*$ & $*$ & $*$ & - \\
$5:$ & $*$ & $*$ & $*$ & $*$ & $*$ & $*$ & $*$ & $*$ & $*$ & $*$ & $*$ & $*$ & $*$ & $*$ & $*$ & - \\
$4:$ & $*$ & $*$ & $*$ & $*$ & $*$ & $*$ & $*$ & $*$ & $*$ & $*$ & $*$ & $*$ & $*$ & $*$ & $*$ & - \\
$3:$ & $*$ & $*$ & $*$ & $*$ & $*$ & $*$ & $*$ & $*$ & $*$ & $*$ & $*$ & $*$ & $*$ & $*$ & $*$ & - \\
$2:$ & $*$ & $*$ & $*$ & $*$ & $*$ & $*$ & $*$ & $*$ & $*$ & $*$ & $*$ & $*$ & $*$ & $*$ & $*$ & - \\
$1:$ & $*$ & $*$ & $*$ & $*$ & $*$ & $*$ & $*$ & $*$ & $*$ & $*$ & $*$ & $*$ & $*$ & $*$ & $*$ & - \\
$0:$ & - & - & - & - & - & - & - & - & - & - & - & - & - & - & - & - \\
\hline
\end{tabular}

Figure 2. The decision matrix for class 1 with $\mathrm{F} 1=500$.

\begin{tabular}{rllllllllllllllll}
\hline $20:$ & $*$ & $*$ & $*$ & $*$ & $*$ & $*$ & $*$ & $*$ & $*$ & $*$ & $*$ & $*$ & $*$ & $*$ & $*$ & 15 \\
19 & $*$ & $*$ & $*$ & $*$ & $*$ & $*$ & $*$ & $*$ & $*$ & $*$ & $*$ & $*$ & $*$ & $*$ & $*$ & - \\
18 & $*$ & $*$ & $*$ & $*$ & $*$ & $*$ & $*$ & $*$ & $*$ & $*$ & $*$ & $*$ & $*$ & $*$ & $*$ & - \\
17 & $*$ & $*$ & $*$ & $*$ & $*$ & $*$ & $*$ & $*$ & $*$ & $*$ & $*$ & $*$ & $*$ & $*$ & $*$ & - \\
$16:$ & $*$ & $*$ & $*$ & $*$ & $*$ & $*$ & $*$ & $*$ & $*$ & $*$ & $*$ & $*$ & $*$ & $*$ & $*$ & - \\
15 & $*$ & $*$ & $*$ & $*$ & $*$ & $*$ & $*$ & $*$ & $*$ & $*$ & $*$ & $*$ & $*$ & $*$ & $*$ & - \\
14 & $*$ & $*$ & $*$ & $*$ & $*$ & $*$ & $*$ & $*$ & $*$ & $*$ & $*$ & $*$ & $*$ & $*$ & $*$ & - \\
13 & $*$ & $*$ & $*$ & $*$ & $*$ & $*$ & $*$ & $*$ & $*$ & $*$ & $*$ & $*$ & $*$ & $*$ & $*$ & - \\
12 & $*$ & $*$ & $*$ & $*$ & $*$ & $*$ & $*$ & $*$ & $*$ & $*$ & $*$ & $*$ & $*$ & $*$ & $*$ & - \\
11 & $*$ & $*$ & $*$ & $*$ & $*$ & $*$ & $*$ & $*$ & $*$ & $*$ & $*$ & $*$ & $*$ & $*$ & $*$ & - \\
10 & $*$ & $*$ & $*$ & $*$ & $*$ & $*$ & $*$ & $*$ & $*$ & $*$ & $*$ & $*$ & $*$ & $*$ & $*$ & - \\
9 & $*$ & $*$ & $*$ & $*$ & $*$ & $*$ & $*$ & $*$ & $*$ & $*$ & $*$ & $*$ & $*$ & $*$ & $*$ & - \\
$8:$ & $*$ & $*$ & $*$ & $*$ & $*$ & $*$ & $*$ & $*$ & $*$ & $*$ & $*$ & $*$ & $*$ & $*$ & $*$ & - \\
$7:$ & - & $*$ & $*$ & $*$ & $*$ & $*$ & $*$ & $*$ & $*$ & $*$ & $*$ & $*$ & $*$ & $*$ & $*$ & - \\
$6:$ & - & - & - & - & $*$ & $*$ & $*$ & $*$ & $*$ & $*$ & $*$ & $*$ & $*$ & $*$ & $*$ & - \\
$5:$ & - & - & - & - & - & - & - & $*$ & $*$ & $*$ & $*$ & $*$ & $*$ & $*$ & $*$ & - \\
$4:$ & - & - & - & - & - & - & - & - & - & - & - & - & - & - & - & - \\
$3:$ & - & - & - & - & - & - & - & - & - & - & - & - & - & - & - & - \\
$2:$ & - & - & - & - & - & - & - & - & - & - & - & - & - & - & - & - \\
$1:$ & - & - & - & - & - & - & - & - & - & - & - & - & - & - & - & - \\
$0:$ & - & - & - & - & - & - & - & - & - & - & - & - & - & - & - & - \\
\hline
\end{tabular}

Figure 3. The decision matrix for class 2 with $F 2=200$. 


\begin{tabular}{|c|c|c|c|c|c|c|c|c|c|c|c|c|c|}
\hline $\begin{array}{r}20: \\
19 \\
18: \\
17 \\
16: \\
15: \\
14 \vdots \\
13: \\
12: \\
11: \\
10: \\
9 \\
8 \\
7 \\
6 \vdots \\
5: \\
4 \vdots \\
3: \\
2 \vdots \\
1: \\
0:\end{array}$ & $\begin{array}{l}= \\
- \\
- \\
= \\
- \\
- \\
- \\
- \\
- \\
- \\
-\end{array}$ & $\begin{array}{l}1 \\
* \\
* \\
*\end{array}$ & $\begin{array}{l}- \\
- \\
- \\
- \\
- \\
-\end{array}$ & $\begin{array}{l}3 \\
* \\
* \\
* \\
* \\
* \\
*\end{array}$ & $\begin{array}{l}\text { * } \\
\text { * } \\
\text { * } \\
\text { * } \\
\text { * } \\
\text { * }\end{array}$ & $\begin{array}{l}* \\
* \\
* \\
* \\
* \\
* \\
* \\
* \\
*\end{array}$ & $\begin{array}{l}* \\
* \\
* \\
* \\
* \\
* \\
* \\
*\end{array}$ & $\begin{array}{l}* \\
* \\
* \\
* \\
* \\
* \\
* \\
*\end{array}$ & H & $\begin{array}{l}* \\
* \\
*\end{array}$ & $\begin{array}{l}* \\
* \\
* \\
*\end{array}$ & $\begin{array}{l}* \\
* \\
* \\
* \\
* \\
* \\
* \\
* \\
* \\
* \\
* \\
- \\
- \\
- \\
- \\
- \\
- \\
- \\
-\end{array}$ & $\begin{array}{l}- \\
= \\
- \\
- \\
- \\
- \\
- \\
- \\
- \\
- \\
- \\
- \\
- \\
- \\
- \\
- \\
-\end{array}$ \\
\hline
\end{tabular}

Figure 4. The decision matrix for class 3 with $F 3=100$.

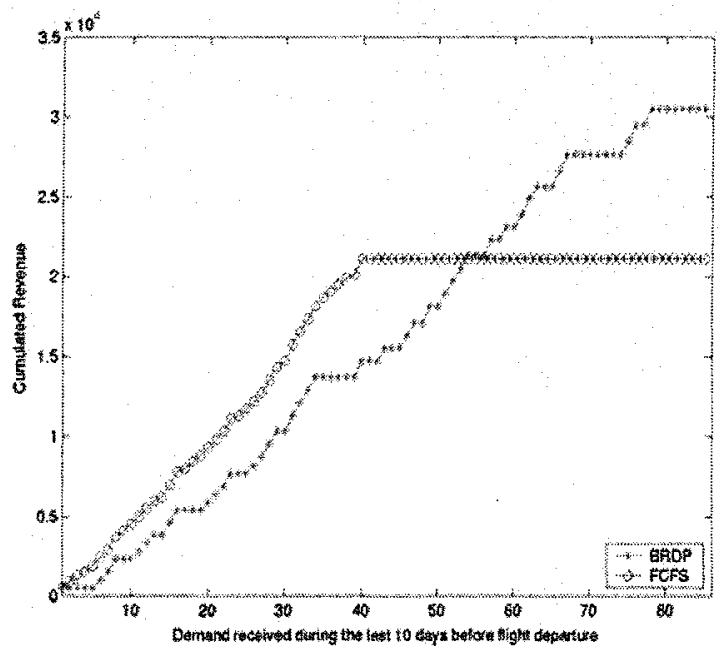

Figure 5. The cumulated actual revenue vs. daily booking requests. 


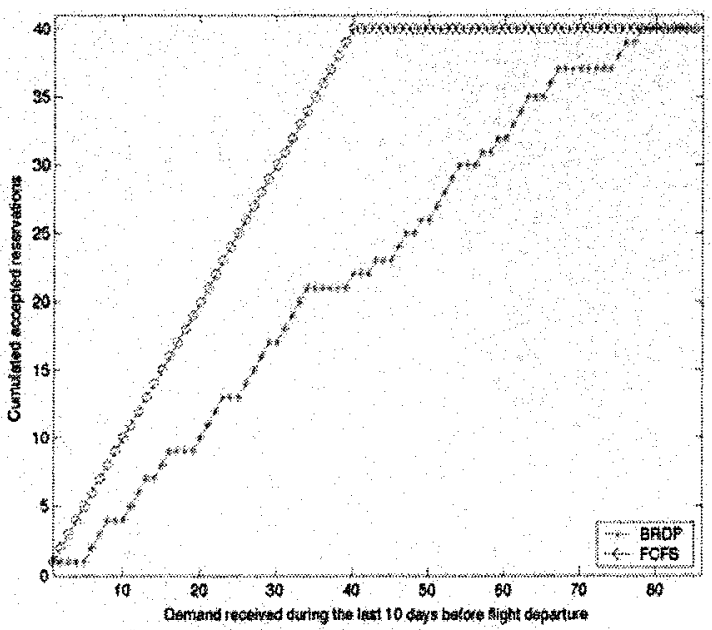

Figure 6 . The cumulated accepted demand vs. daily booking requests

\section{CONCLUSIONS}

In this communication a new methodology based on dynamic programming has been proposed to establish an on-line airline inventory control process. This approach presents characteristics which turn it of real interest for practical utilization by airlines.

It is clear that, here again, the performance of the proposed control process depends on the quality of the available estimations of demand distributions. However, the recursive characteristic of the proposed optimisation method makes it fully compatible with an on-line demand estimation process which takes advantage of the newest available information.

Taking into account the simulation results obtained, presented in Section 4 , good performances of the proposed methodology, when applied within a real system, are expected. 


\section{REFERENCES}

Bellman, R., 1957, Applied Dynamic Programming. Princeton University Press, Princeton, N. J.

Belobaba, P.P., 1989, Application of a probabilistic decision model to airline seat inventory control. Operations Research, 37, pp. 183-197.

Bertsekas, D.P., 2000, Dynamic programming and Optimal Control, Volume I: second edition, Athena Scientific, Belmont, Massachusetts.

Bertsekas, D.P., 2001, Dynamic programming and Optimal Control, Volume II: second edition, Athena Scientific, Belmont, Massachusetts.

Bilegan, I.C., W. El Moudani, A. Achaibou and F. Mora-Camino, 2001, A new approach to update probability distributions estimates of air travel demand. Smart Engineering System Design, 11, pp. 853-856.

Fabrycky, W.J. and P.E. Torgersen, 1966, Operations Economy - Industrial Applications of Operations Research. Prentice-Hall, Inc., Englewood Cliffs, N. J.

Gerchak, Y., M. Parlar and T.K.M. Yee, 1985, Optimal Rationing Policies and Production Quantities for Products with Several Demand Classes. Can. J. Admin. Sci., 2, pp 161-176.

Goldberg, D.E., 1989, Genetic Algorithms in Search, Optimization and Machine Learning. Addison-Wesley.

Hillier, F.S. and G.J. Lieberman, 1967, Introduction to Operations Research. Holden-Day, Inc., San Francisco.

Jumarie, G.R.M., 1990, Relative Information: Theories and Applications. Springer Verlag.

Lee, T.C. and M. Hersh, 1993, A Model for Dynamic Airline Seat Inventory Control with Multiple Seat Bookings. Transportation Science, 27, pp. 252-265.

Littlewood, K., 1972, Forecasting and Control of Passenger Bookings. AGIFORS Proceedings, 12, pp. 95-117.

McGill, J.I. and G.J. van Ryzin, 1999, Revenue Management: Research Overview and Prospects. Transportation Science, 33, pp. 233-256.

Mora-Camino, F., 1978, Introduction à la Programmation Géométrique. COPPE, Rio de Janeiro.

Subramanian, J., 1995, Airline Yield Management and Computer Memory Management via Dynamic Programming. Ph.D. Dissertation, University of North Carolina, Dept. of Operations Research, Chapel Hill, N. C.

Talluri K. and G.J. van Ryzin, 1998, An Analysis of Bid-Price Controls for Network Revenue Management. Management Science, 44, pp. 1577-1593.

Winston, W.L., 1994, Operations Research - Applications and Algorithms: third edition. Duxbury Press, Belmont, California. 